\title{
Comprehensive nationwide analysis of mother-to-child HIV transmission in Finland from 1983 to 2013
}

\section{Original Paper}

Cite this article: Aho I, Kivelä P, Kaijomaa M, Surcel H-M, Ristola M, Heikinheimo O, Sutinen J, with the FINHIVPREG Study Team (2018). Comprehensive nationwide analysis of mother-to-child HIV transmission in Finland from 1983 to 2013. Epidemiology and Infection 146, 1301-1307. https://doi.org/10.1017/ S0950268818001280

Received: 6 February 2018

Revised: 22 March 2018

Accepted: 24 April 2018

First published online: 15 May 2018

\section{Key words:}

Epidemiology; HIV/AIDS; prevention

Author for correspondence:

Inka Aho, E-mail: inka.aho@hus.fi

\section{Aho' ${ }^{1}$, P. Kivelä1 ${ }^{1}$ M. Kaijomaa², H.-M. Surcel ${ }^{3}$, M. Ristola ${ }^{1}$, O. Heikinheimo ${ }^{2}$, J. Sutinen ${ }^{1}$ and with the FINHIVPREG Study Team}

${ }^{1}$ Department of Infectious Diseases, Helsinki University Hospital and University of Helsinki, Finland; ${ }^{2}$ Department of Obstetrics and Gynecology, Helsinki University Hospital and University of Helsinki, Finland and ${ }^{3} \mathrm{National}$ Institute of Health and Welfare, Oulu and Faculty of Medicine, University of Oulu, Finland

\begin{abstract}
HIV-positive children are still born in Europe despite low mother-to-child transmission (MTCT) rates. We aimed to clarify the remaining barriers to the prevention of MTCT. By combining the national registers, we identified all women living with HIV delivering at least one child during 1983-2013. Of the 212 women delivering after HIV diagnosis, 46\% were diagnosed during the pregnancy. In multivariate analysis, age $>30$ years $(P=0.001)$, sexual transmission $(P=0.012)$, living outside of the metropolitan area $(P=0.001)$ and Eastern European origin $(P=0.043)$ were risk factors for missed diagnosis before pregnancy. The proportion of immigrants increased from $18 \%$ before 1999 to $75 \%$ during $2011-2013(P<0.001)$. They were diagnosed during the pregnancy equally to natives and achieved similar, good treatment results. No MTCT occurred when the mother was diagnosed before the delivery. In addition, 12 women had delivered in 2 years prior their HIV diagnosis, most before implementation of the national screening of pregnant women. Three of these children were infected, the last one in 2000. Our data demonstrate that complete elimination of MTCT is feasible in a high-income, low-prevalence country. This requires ongoing universal screening in early pregnancy and easy access to antiretroviral therapy to all HIV-positive people.
\end{abstract}

\section{Introduction}

After widespread implementation of antiretroviral therapy (ART), several studies from European countries have reported very low mother-to-child transmission (MTCT) rates of HIV [1-4]. Despite these successes, approximately 200-300 HIV-positive children are reported to the European Centre for Disease Prevention and Control (ECDC) annually. Approximately half of these children are immigrants and are, most probably, infected already in their country of origin [5]. To confirm MTCT as the route of transmission, one should know the mother's HIV status at the time of the delivery. This is, unfortunately, known in only a limited number of cases.

The proportion of immigrants of all HIV-positive patients is increasing in Europe. There is a concern that eliminating MTCT is less successful among immigrant women, e.g. a Canadian study showed that immigrant HIV-positive women received less adequate prenatal care compared with Canadian women [6]. Likewise there appears to be barriers to testing and care also in Europe [7].

Elimination of MTCT requires a bundle of interventions: ART to the mother and the newborn, appropriate management of delivery and avoidance of breastfeeding. Since only diagnosed women can be treated, the cornerstone in preventing MTCT is timely HIV diagnosis. Women should be diagnosed in early antenatal care if not diagnosed before conception.

When all precautions are met, even zero transmissions can be achieved in a subgroup of women on ART at the time of conception, as reported by Mandelbrot et al. [8]. However, we are unaware of data on eliminating MTCT on a national level nor on women delivering with undiagnosed HIV.

Finland implemented a universal opt-out antenatal HIV screening in 1998. Several cities had already screened pregnant women for some years. Approximately $98 \%$ of all pregnant women in Finland attend this screening [9]. With the approval of ethical committees for research purposes, the health care registers in Finland permit individual identification of all HIV-positive women giving birth either before or after their own HIV diagnosis.

The aim of the main study was to determine the national MTCT rate in Finland since the beginning of the HIV epidemic. We also evaluated the risk factors leading to late diagnosis of HIV during pregnancy and compared the diagnostics and treatment results between natives and immigrants. As a substudy, we reviewed women that delivered $<2$ years before their 
HIV diagnosis, analysed the HIV status of these children and sought to clarify the reasons leading to unknown HIV status before the delivery.

\section{Methods}

In the main study, we included all HIV-positive women with at least one delivery in Finland during 1983-2013 after receiving HIV diagnosis and all children born in these deliveries. In a substudy, we included women who had a delivery within 2 years prior to HIV diagnosis and whose HIV diagnosis was unknown at the time of the delivery. The year 1983 appeared to be the starting point as the first year a woman was diagnosed HIV-positive in Finland [10]. To allow adequate follow-up of the children, we chose 31 December 2013 as the closing point.

In Finland, each individual receives a unique, 10-digit personal identification number at birth or immigration by the Civil Registration System. With this number, a person can be identified in different registers and hospitals' medical records throughout the country.

Laboratories report each individual's first positive HIV antibody test result to the National Infectious Diseases Register maintained by the National Institute of Health and Welfare [10]. Physicians report detailed information on the mode of transmission of HIV, nationality, country of transmission and stage of the disease to the registry.

Hospitals report all children born in Finland to the Medical Birth Register regardless of mother's nationality [11]. This register contains information on the mother, the delivery and the newborn from 1987 onwards. We collected the treatment/delivery data from 1983 to 1987 from the hospitals' medical records.

The Finnish Maternity Cohort Register contains information on antenatal infection screening results from 1998 onwards [12] and was used to complete and verify the data.

HIV-positive patients are treated in Infectious Diseases Clinics in 20 hospitals in Finland; 16 of them also manage deliveries of HIV-positive women.

We combined the National Infectious Diseases Register with Medical Birth Register and Finnish Maternity Cohort Register to identify all HIV-positive women ever delivering in Finland.
Their children were identified from these registries. Data on women and children were collected from the registers as well as from the hospitals' medical records (Fig. 1). The study subjects were not contacted.

We extracted the following data on women of known HIV diagnosis at the time of the delivery: year, country, site of HIV diagnosis, mode of transmission, possible previous AIDS defining illnesses, hepatitis B surface antigen (HBsAg) status, hepatitis C antibodies and number of children and induced abortions prior to HIV diagnosis, as well as country of origin and year of immigration. We collected the following data for each pregnancy: ART status before the pregnancy and during each trimester, CD4 lymphocyte count at the beginning of the pregnancy, HIV viral load (VL) preceding the delivery, HIV status of the newborn and information on previous children born in Finland and their HIV status.

For women who had given birth within 2 years prior to their own HIV diagnosis, we collected the following data: year and hospital of the delivery, country of origin, time of the HIV diagnosis related to the delivery and possible explanation of why HIV test was not taken before the delivery. For children born before the mother's HIV diagnosis, only HIV status was collected.

The ethics committee of Helsinki University Hospital approved the study (344/13/03/00/2014). The National Institute of Health and Welfare granted permission to combine the registers and to perform the nationwide study (THL/1535/6.02.00/ 2015). All 16 participating hospitals provided local permissions to carry out the study and to use their medical records. According to the Finnish legislation, informed consent is not required for this type of a retrospective study.

\section{Statistical analysis}

HIV diagnosis was classified as before pregnancy if the diagnosis had been made before conception. HIV diagnosis after gestational week (GW) 20 was considered very late as was the initiation of ART after GW 24.

The detection limit of VL measurement declined during the study years from 1000 to 20 copies/mL. We grouped all undetectable VL results with detectable VL $<200$ copies $/ \mathrm{mL}$ as a good
Fig. 1. Combining the registers to identify all HIV-related pregnancies and the HIV status of the children. Two hundred and twelve women were diagnosed HIV-positive before or during the pregnancy. Twelve women were diagnosed HIV-positive $<2$ years after a delivery with an unknown HIV status at the time of the delivery.

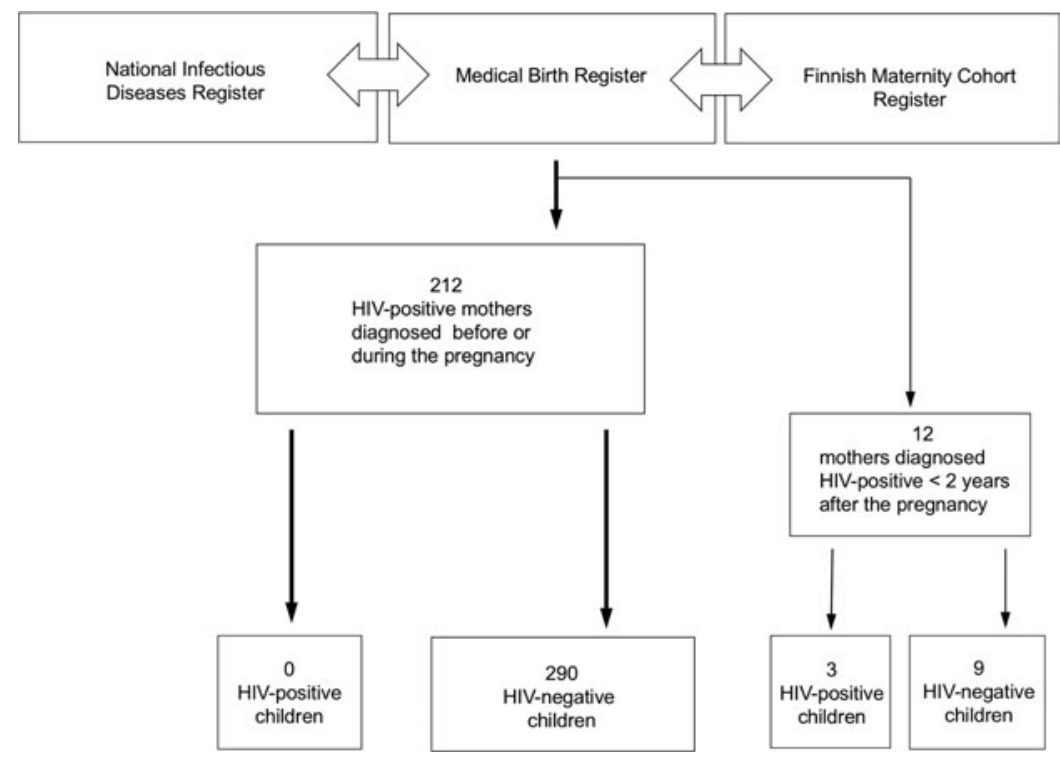


treatment response. Detectable VL exceeding 200 copies $/ \mathrm{mL}$ preceding the delivery was considered an inadequate treatment response.

For comparisons between the groups, $\chi^{2}$ and Fisher's exact test were used for categorical variables and the non-parametric Mann-Whitney $U$-test for continuous variables. We used univariate and multivariate logistic regression models to evaluate risk factors for HIV diagnosis during the pregnancy as compared with diagnosis before the pregnancy.

IBM SPSS version 21.0 (Chicago, IL, USA) was used in all statistical analyses.

\section{Results}

During 1983-2013, altogether 212 women with HIV diagnosis gave birth to 290 children. Helsinki University Hospital accounted for $65.2 \%$ of these deliveries. The first delivery occurred in 1993. The prevalence of diagnosed HIV among parturients increased from 3.1/100 000 in 1993 to 65.8/100 00 in 2013.

The population of $212 \mathrm{HIV}$-positive women consisted of altogether 138 (65.1\%) immigrants and 74 (34.9\%) native women. Before the index delivery, 38.2\% $(n=81)$ of the women had children and $30.7 \%(n=65)$ had undergone an induced abortion. The proportion of women with previous children was higher in immigrants compared with natives $(44.2 \%$ vs. $27.0 \%, P=$ $0.014)$. After the HIV diagnosis, $36.8 \%(n=78)$ of the population had more than one child with no difference between immigrants and natives (Table 1).

Before the index delivery, $3.8 \%$ had a preceding AIDS-defining illness, $4.7 \%$ were $\mathrm{HBsAg}$-positive and $10.8 \%$ hepatitis $\mathrm{C}$ virusantibody positive. After the index delivery, there were no cases of new AIDS-defining illnesses or hepatitis seroconversions.

The proportion of immigrants among the HIV-positive parturients increased significantly from $18.2 \%$ before 1999 to $75.3 \%$ during 2011-2013 $(P<0.001)$. The highest increase was found in women born in sub-Saharan Africa and in Eastern Europe (Fig. 2).

\section{Factors associated with HIV diagnosis during the pregnancy}

When analysing the risk factors for HIV diagnosis during pregnancy, we included only the first pregnancy as HIV-positive. Of all 212 women, $45.8 \%$ were diagnosed during pregnancy. If all 290 pregnancies were included, the proportion of women diagnosed during the pregnancy decreased to $34.1 \%$. The proportion of women diagnosed during the pregnancy did not change with time nor did the proportion diagnosed very late after GW 20 .

The HIV diagnosis at the antenatal screening programme was more common outside the Helsinki metropolitan area than in Helsinki $(65.2 \%$ vs. $35.8 \%, P<0.001)$ (Fig. 3). The proportion of immigrant and native women diagnosed during pregnancy did not differ significantly (53.0\% vs. $42.3 \%, P=0.153)$.

In multivariate analyses, women aged over 30 years, heterosexual route of transmission compared with intravenous drug use, living outside of the Helsinki metropolitan area and being of Eastern European origin were risk factors for being diagnosed during pregnancy (Table 2). When assessing the risk factors for very late diagnosis, immigrants were more often diagnosed after 20 GWs compared with natives $(13.0 \%$ vs. $2.7 \%, P=0.014)$ and with CD4 counts $<200$ cells $/ \mu \mathrm{L}(16.4 \%$ vs. $1.5 \%, P=0.002)$. When a higher CD4 cut-off of 350 cells/ $\mu \mathrm{L}$ was used, immigrants and natives did not differ significantly $(45.0 \%$ vs. $29.6 \%, P=$ $0.176)$.
Table 1. Demographics of the 212 women delivering at least one child after HIV diagnosis 1983-2013

\begin{tabular}{|c|c|}
\hline Age & Median (IQR) \\
\hline At HIV diagnosis & $26(23,30)$ \\
\hline At first HIV pregnancy & $30(26,34)$ \\
\hline Time from dg to delivery (years) & $1(0,4)$ range $0-16$ \\
\hline \multirow{2}{*}{$\begin{array}{l}\text { Time from immigration to diagnosis of } \\
\text { immigrants diagnosed in Finland }(n=115)\end{array}$} & $1(0,4)$ range $0-15$ \\
\hline & $n(\%)$ \\
\hline \multicolumn{2}{|l|}{ Origin } \\
\hline Finland & $74(34.9)$ \\
\hline Eastern Europe & $31(14.6)$ \\
\hline Western Europe and America & $1(0.5)$ \\
\hline Sub-Saharan Africa & $73(34.4)$ \\
\hline Asia & $33(15.6)$ \\
\hline \multicolumn{2}{|l|}{ Mode of transmission } \\
\hline Sex & $179(84.4)$ \\
\hline IDU & $19(9.0)$ \\
\hline MTCT & 0 \\
\hline Other/not known & $14(6.6)$ \\
\hline \multicolumn{2}{|l|}{ First CD4 count during pregnancy cells $/ \mu \mathrm{L}$} \\
\hline$<200$ & $23(10.8)$ \\
\hline $200-349$ & $38(17.9)$ \\
\hline $350-500$ & $57(26.9)$ \\
\hline$>500$ & $84(39.6)$ \\
\hline Missing data & $10(4.7)$ \\
\hline \multicolumn{2}{|l|}{ Time of the diagnosis } \\
\hline Before pregnancy & $115(54.2)$ \\
\hline During pregnancy, $\mathrm{GW} \leqslant 20$ & $77(36.3)$ \\
\hline During pregnancy, GW>20 & $20(9.4)$ \\
\hline \multicolumn{2}{|l|}{$\begin{array}{l}\text { Number of induced abortions before first } \\
\text { delivery as HIV-positive }\end{array}$} \\
\hline 0 & $147(69.3)$ \\
\hline 1 & $37(17.5)$ \\
\hline 2 & $23(10.8)$ \\
\hline 3 or more & $5(2.3)$ \\
\hline \multicolumn{2}{|l|}{ Country of HIV diagnosis of immigrants $(N=138)$} \\
\hline Finland & $115(83.3)$ \\
\hline Original home country & $17(12.3)$ \\
\hline Other country & $6(4.3)$ \\
\hline
\end{tabular}

IQR, interquartile range; IDU, intravenous drug use; MTCT, mother-to-child HIV transmission; $\mathrm{GW}$, gestational week

\section{Antiretroviral treatment}

If assessing all 290 pregnancies, $53.9 \%$ of mothers were on ART at the time of conception with no difference between immigrants and natives $(55.6 \%$ vs. $51.4 \%, P=0.570)$. The proportion of women on ART at the time of conception was higher in 20102013 compared with earlier years $(54.4 \%$ vs. $45.6 \%, P=0.001)$. 
Fig. 2. The number and the origin of the parturients according to the year of delivery. The number of immigrants among HIV-positive parturients increased over fourfold between 1999 and 2013.

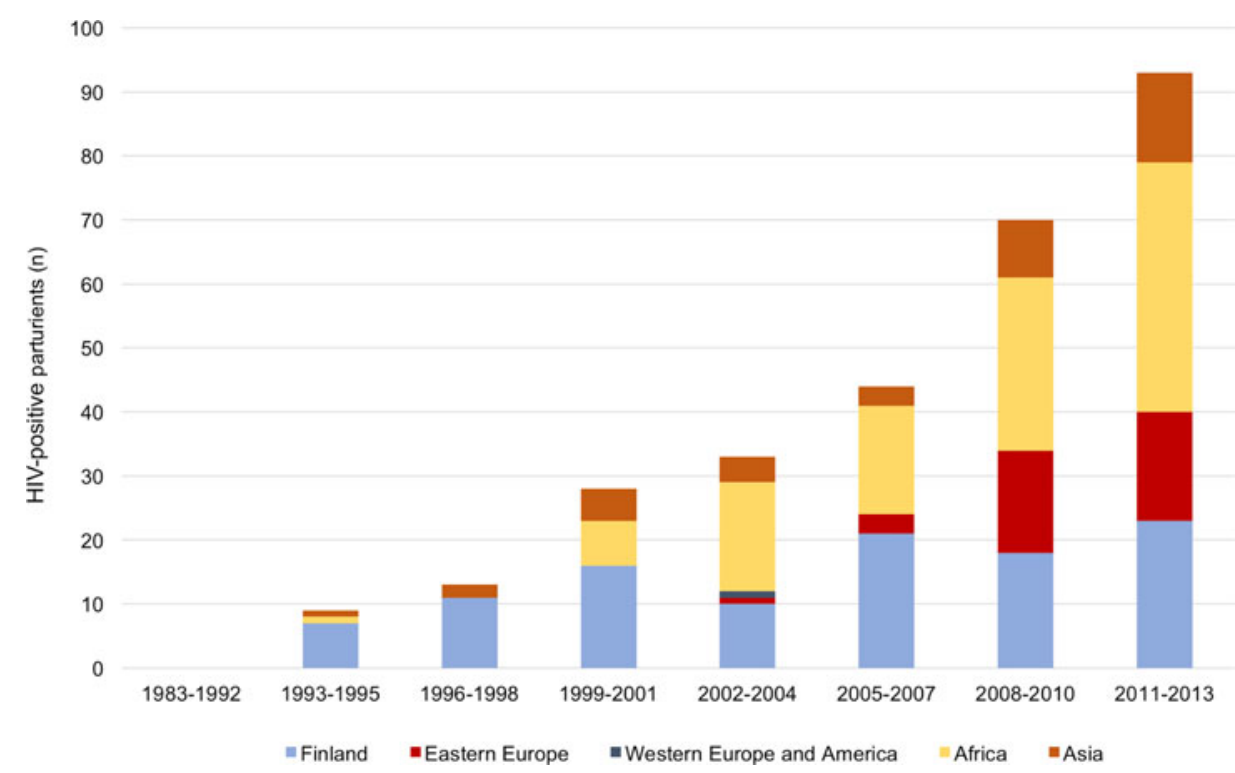

An increasing proportion of women were on ART by GW 24 (Fig. 4) over time with no difference between immigrants and natives $(74.5 \%$ vs. $73.6 \%, P=0.870)$. All women were prescribed ART in the third trimester. Six women refused to start ART with no difference between immigrants and natives.

The proportion of women with a good treatment response to ART (VL preceding the delivery undetectable or $<200$ copies $/ \mathrm{ml}$ ) increased during the study period (Fig. 4). We found no difference in the proportion of women with a good treatment response to ART between immigrants and natives $(85.9 \%$ vs. $82.1 \%, P=$ 0.390 ), or between the Helsinki metropolitan area and the rest of Finland (84.1\% vs. $85.1 \%, P=0.819)$.

\section{MTCT of HIV during 1983-2013}

Of the 290 children born to mothers with diagnosed HIV infection, none were infected.

During the study period, altogether 12 pregnant women had an unknown HIV status during the delivery and were diagnosed within 2 years afterwards (Fig. 1). Eight deliveries occurred before implementation of the national screening programme in 1998, three immigrant women did not attend the screening and one native woman was infected during the pregnancy after a negative test result in early pregnancy. Three (i.e. 25\%) children of these undiagnosed mothers were infected, the last one in 2000.

\section{Discussion}

The present study shows that elimination of MTCT of HIV is feasible in a low-prevalence, high-resource country. No child has been infected perinatally with HIV in Finland since the year 2000, even though the number of deliveries in HIV-positive women has increased and a high proportion (46\%) of them continue to be diagnosed first during pregnancy. Moreover, the proportion of HIV diagnoses made during pregnancy did not decrease.

Older age, living outside the Helsinki area, Eastern European origin and not being infected by drug use were associated with an increased risk of being diagnosed only during pregnancy. Intravenous drug users in Finland are actively offered lowthreshold HIV testing and the infections have been diagnosed early [13], which probably explains this surprising result concerning drug use in preventing late diagnosis. Moreover, most women of Eastern European and South-East Asian origin arrive in Finland to work, or as spouses, and thus are not tested routinely, unlike refugees and asylum seekers.

Several recent publications report a substantially lower number of mothers diagnosed with HIV in the pregnancy, for example, from Denmark (18\%), Germany (24\%) and the UK (28\%) [1, 3, $14]$, but in these articles, all pregnancies were included and multiparity obviously decreases the figures. We only included each woman's first pregnancy after the HIV diagnosis in our analyses, considering this the most relevant. In our study, the rate of
Fig. 3. Site of diagnosis in the Helsinki metropolitan area and outside the metropolitan area. Altogether $46 \%$ of the parturients were diagnosed during pregnancy with a significant difference between Helsinki metropolitan area and the rest of Finland.

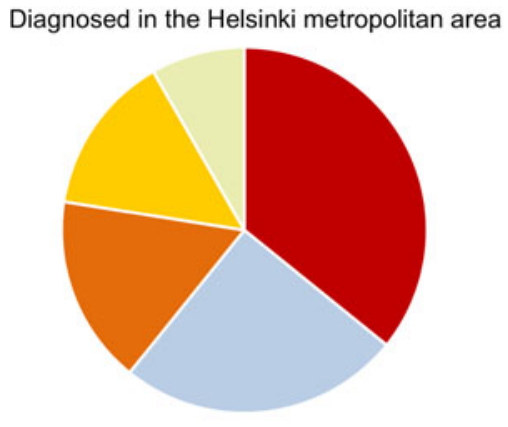

- Antenatal care
Diagnosed outside the metropolitan area

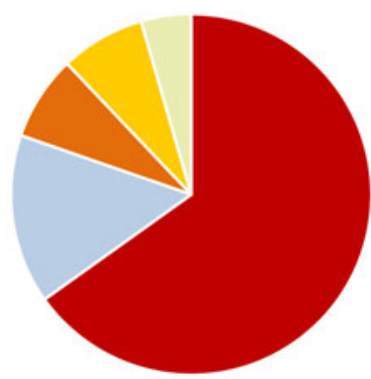

= Immigrant services 
Table 2. Risk factors for not being diagnosed before the pregnancy for women diagnosed in Finland $(N=186)$

\begin{tabular}{|c|c|c|c|c|c|c|}
\hline & Univariate OR & $95 \% \mathrm{Cl}$ & $P$-value & Multivariate $\mathrm{OR}$ & $95 \% \mathrm{Cl}$ & $P$-value \\
\hline \multicolumn{7}{|l|}{ Age (years) } \\
\hline$\leqslant 30$ & 1.00 & & & & & \\
\hline$>30$ & 3.57 & $1.70-7.52$ & 0.001 & 3.86 & $1.70-8.76$ & 0.001 \\
\hline \multicolumn{7}{|l|}{ Region of origin } \\
\hline Africa & 1.17 & $0.59-2.30$ & 0.655 & 1.17 & $0.53-2.61$ & 0.699 \\
\hline Asia & 1.87 & $0.78-4.50$ & 0.159 & 1.99 & $0.74-5.34$ & 0.174 \\
\hline \multicolumn{7}{|c|}{ Diagnosis in Helsinki metropolitan area } \\
\hline Yes & 1.0 & & & & & \\
\hline Sexual & 7.69 & $1.70-34.88$ & 0.008 & 8.17 & $1.58-42.31$ & 0.012 \\
\hline Year of the delivery & 0.99 & $0.95-1.05$ & 0.970 & 0.96 & $0.91-1.03$ & 0.255 \\
\hline
\end{tabular}

$\mathrm{OR}$, odds ratio; $\mathrm{Cl}$, confidence interval; IDU, intravenous drug use.

${ }^{a}$ Natives and one immigrant of Western European origin were combined as Western European origin.

diagnosis during pregnancy was $34 \%$ if all pregnancies were included; this is still higher than published elsewhere. Favarato et al. concluded that pregnancy is an important opportunity to migrant women to learn their HIV status [15]. Our study in a very-low-prevalence setting extends this conclusion also to local women, since both groups were equally undiagnosed before the pregnancy. About half of the women diagnosed at antenatal screening had no obvious risk factor for HIV infection and could have been left out in a risk-based screening. Before the implementation of the universal antenatal screening in 1998, the mother was undiagnosed in 8/30 (27\%) of HIV-related deliveries. The same was true in only $4 / 272(1.5 \%)$ deliveries after 1998. Taken together, these observations highlight the continuous need for systematic antenatal HIV screening, even in lowprevalence countries.

Notably, 30\% of women had had an induced abortion before the first pregnancy as HIV-positive. Although we were unable to specify whether they were already HIV-positive at the time of the abortion, it could serve as a valuable testing opportunity.
Treatment of HIV is not centralised in Finland, but the Helsinki area accounted for $65 \%$ of HIV-related pregnancies. Women living outside the Helsinki metropolitan area were more often diagnosed late than women living in the Helsinki area. There was no difference, however, in treatment response between Helsinki and the other areas, even the smallest hospitals. This is reassuring, since long geographical distances hinder centralisation of HIV care. Earlier studies have shown discrepant findings in this respect: an increased risk of late diagnosis in people living in low-prevalence areas was shown in non-pregnant patients in Spain and the UK $[16,17]$. In contrast, in a recent study from the UK, women living outside the London area had lower odds on late booking to antenatal care compared with women living in London [18]. This study describes the delays in diagnostics and treatment initiation, but lacks information on treatment response.

The proportion of immigrants among HIV-positive parturients increased in our study although later and to a lesser extent than in many other European countries [15]. Women originating

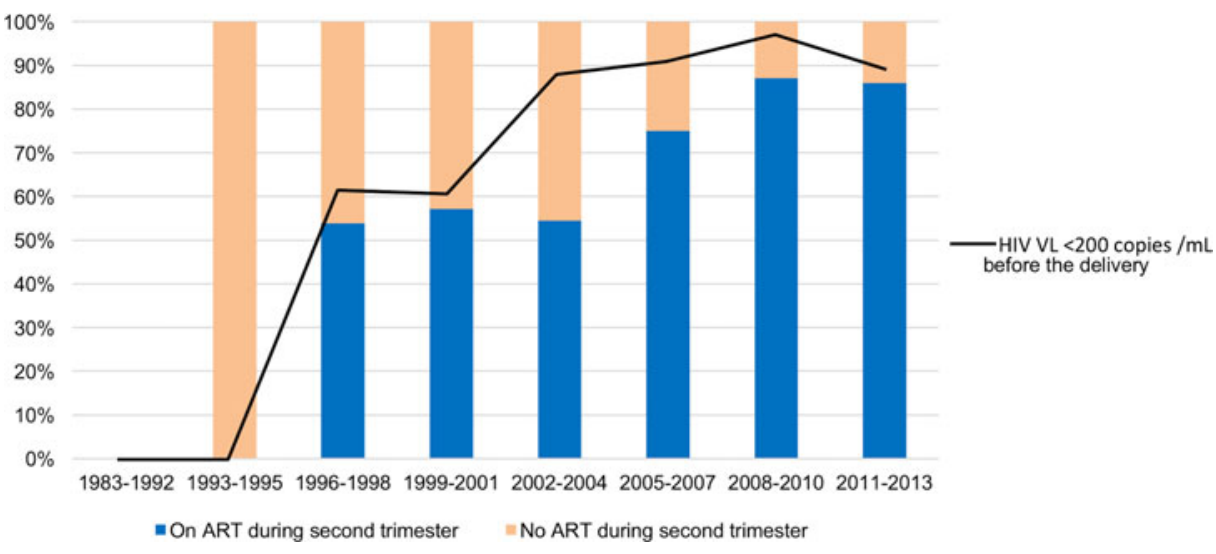

Fig. 4. The proportion of the parturients on antiretroviral therapy (ART) during the second trimester (bars) and with HIV viral load (VL) $<200$ copies/mL preceding the delivery (line) over the study period. 
from sub-Saharan Africa formed the largest group, but the proportions of women from South-East Asia and Eastern Europe were substantial, unlike in many other countries.

The proportion of women with very late diagnosis (after GW 20) and with very low CD4 counts ( $<200$ cells/ $\mu \mathrm{L})$ at diagnosis were significantly higher among immigrants than natives. The difference in very late diagnosis was mainly driven by women from sub-Saharan Africa arriving in Finland late in pregnancy. Special multidisciplinary attention should be taken to reach these women as soon as possible after their immigration. Our figures of very late diagnosis were nonetheless substantially lower than in a recent European cohort study [15]. In that study, immigrants were more often diagnosed during pregnancy, diagnosed late in pregnancy and with a lower CD4 count than local women. In our study, the proportions of diagnosis during earlier in the pregnancy, ART coverage during the second trimester, virological treatment results and proportion on ART at the time of conception were similar between these groups. In a recent study from the UK [18], immigrants, especially from sub-Saharan Africa, booked late for an antenatal visit and those newly diagnosed started ART later than natives, which is in contrast to our results.

In our study, only half of the previously diagnosed women were on ART at the time of conception with no differences between immigrants and natives. Most of the study period occurred before the 2012 WHO recommendation that women should stay on ART after the delivery [19]. The proportion on ART increased worldwide after this recommendation and has further increased after the results of the START study [20] showed ART to be beneficial to all HIV-positive individuals, regardless of the CD4 count. According to present guidelines, all women are recommended to continue lifelong ART after the delivery. This may reduce the number of loss to follow-up, since not being on ART seems to increase that risk [21].

Most women, whether diagnosed before or during the pregnancy, were on ART by GW 24, the proportion reaching $87 \%$ after 2008. A great majority of women achieved good virological response, similar to most recently published studies $[1,3,14]$. Even though ART is free of charge for all pregnant women, not everyone agreed to start it, which leaves their children with an excess risk of transmission. The hesitation of these women should be addressed as early as possible with a multidisciplinary approach.

The generalisability of our study results is limited by the relatively low number of HIV-related pregnancies in Finland, even when taking the first 30 years of the epidemic into account. Due to the retrospective nature, we were able to use only data collected at the time. However, we believe our data are complete, since we were able to identify all HIV-related pregnancies by combining the nationwide registers, and including pregnancies and their outcomes prior to women's own HIV diagnosis. In addition, we had access to actual patients' medical records, so we were not dependent on the information collected by the registers only.

By limiting the data collection to 2 years prior to each woman's HIV diagnosis, we might have underestimated the number of HIV-related pregnancies. Since the Medical Birth Register starts from 1987, we may have missed a HIV-related pregnancy during 1983-1987, with 11 women diagnosed during that time. Nevertheless, no HIV-positive children were born in Finland during those years. With a high level of certainty, we can be sure to have included all HIV-positive children born 1983-2013, since no new HIV diagnosis among children born in Finland have been reported by November 2017 .
In conclusion, our study has demonstrated that national elimination of MTCT is feasible in a high-income, low-prevalence country. The mainstay of this success is to enable all women (and men) in their fertile age to know their HIV status in early antenatal screening - although preferably already before the conception - and to reassure HIV-positive women on the safety and effectiveness of ART also during pregnancy.

Acknowledgements. Members of the FINHIVPREG study team: Inka Aho, Helsinki University Hospital; Timo Hautala, Oulu University Hospital, Oulu; Taru Finnilä, Turku University Hospital, Turku; Hanna Viskari, Tampere University Hospital, Tampere; Janne Mikkola, Kanta-Häme Hospital District, Hämeenlinna; Sari Hämäläinen, Kuopio University Hospital, Kuopio; Tuomas Nieminen, Satakunta Central Hospital, Pori; Elina Kärnä, Seinäjoki Central Hospital, Seinäjoki; Ville Lehtinen, Päijät-Häme Central Hospital, Lahti; Maija Rummukainen, Central Finland Health Care District, Jyväskylä; Antti Väänänen, Lapland Central Hospital, Rovaniemi; Jukka Heikkinen, North Carelia Central Hospital, Joensuu; Risto Pietikäinen, Kymenlaakso Central Hospital, Kotka; Pekka Suomalainen, South Carelia Central Hospital, Lappeenranta; Sakari Vuorinen, Mikkeli Central Hospital, Mikkeli. The authors would like to thank Mikko Lehtovirta for the technical assistance with the figures.

Financial support. This work was supported by the Finnish Medical Association (IA) and Infektiotautien tutkimusyhdistys ry (IA).

Conflict of interest. None.

\section{References}

1. Reitter A et al. (2014) Pregnancy complications in HIV-positive women: 11-year data from the Frankfurt HIV Cohort. HIV Medicine 15, 525-536.

2. von Linstow ML et al. (2010) Prevention of mother-to-child transmission of HIV in Denmark, 1994-2008. HIV Medicine 11, 448-456.

3. Townsend CL et al. (2014) Earlier initiation of ART and further decline in mother-to-child HIV transmission rates, 2000-2011. AIDS (London, England) 28, 1049-1057.

4. Warszawski J et al. (2008) Mother-to-child HIV transmission despite antiretroviral therapy in the ANRS French Perinatal Cohort. AIDS (London, England) 22, 289-299.

5. European Centre for Disease Prevention and Control (2016) Annual Epidemiological Report 2016 - HIV/AIDS. Stockholm: ECDC. Available at http://ecdc.europa.eu/en/healthtopics/aids/surveillancereports/pages/ AnnualEpidemiologicalReport2016. Accessed 17 August 2017.

6. Ng R et al. (2015) Adequacy of prenatal care among women living with human immunodeficiency virus: a population-based study. BMC Public Health 15, 514. doi: 10.1186/s12889-015-1842-y.

7. Hernando V et al. (2015) HIV infection in migrant populations in the European Union and European Economic Area in 2007-2012: an epidemic on the move. Journal of Acquired Immune Deficiency Syndromes 70, 204-211.

8. Mandelbrot L et al. (2015) No perinatal HIV-1 transmission from women with effective antiretroviral therapy starting before conception. Clinical Infectious Diseases 61, 1715-1725.

9. Surcel $\mathbf{H}$ et al. Alkuraskauden infektioseulonta Suomessa. Raportti/ Terveyden ja hyvinvoinnin laitos $($ THL $)=$ Report/National Institute for Health and Welfare 2014; (7). Available at http://urn.fi/URN:ISBN:978952-302-057-3. Accessed 18 August 2017.

10. National Infectious Diseases Register (2017) Available at https://www.thl. fi/ttr/gen/rpt/hivsuo.pdf. Accessed 14 March 2017.

11. Perinatal statistics - parturients, deliveries and newborns (2015). Available at http://urn.fi/URN:NBN:fi-fe2017103150386. Accessed 2 January 2018.

12. FMC-rekisteri (2017) Available at https://www.thl.fi/fi/tutkimus-ja-asian tuntijatyo/hankkeet-ja-ohjelmat/finnish-maternity-cohort-fmc-seerumi pankki. Accessed 10 May 2017. 
13. Kivela PS et al. (2010) Determinants of late HIV diagnosis among different transmission groups in Finland from 1985 to 2005. HIV Medicine 11, 360-367.

14. Orbaek M et al. (2017) Assessment of mode of delivery and predictors of emergency caesarean section among women living with HIV in a matched-pair setting with women from the general population in Denmark, 2002-2014. HIV Medicine 18, 736-747. doi: 10.1111/hiv.12519.

15. Favarato G et al. (2018) Migrant women living with HIV in Europe: are they facing inequalities in the prevention of mother-to-child-transmission of HIV? The European Pregnancy and Paediatric HIV Cohort Collaboration (EPPICC) study group in EuroCoord. European Journal of Public Health 28, 55-60. doi: 10.1093/eurpub/ckx048.

16. Castilla J et al. (2002) Late diagnosis of HIV infection in the era of highly active antiretroviral therapy: consequences for AIDS incidence. AIDS (London, England) 16, 1945-1951.
17. Chadborn TR et al. (2005) No time to wait: how many HIV-infected homosexual men are diagnosed late and consequently die? (England and Wales, 1993-2002). AIDS (London, England) 19, 513-520.

18. French CE et al. (2017) Presentation for care and antenatal management of HIV in the UK, 2009-2014. HIV Medicine 18, 161-170.

19. World Health Organization, Geneva (2012) Use of antiretroviral drugs for treating pregnant women and preventing HIV infection in infants. April 2012. Available at http://www.who.int/hiv/PMTCT_update.pdf. Accessed 17 August 2017.

20. Lundgren JD et al. (2015) Initiation of antiretroviral therapy in early asymptomatic HIV infection. New England Journal of Medicine 373, 795-807.

21. Gerver SM et al. (2010) High rate of loss to clinical follow up among African HIV-infected patients attending a London clinic: a retrospective analysis of a clinical cohort. Journal of the International AIDS Society 13, 29. doi: 10.1186/1758-2652-13-29. 